\title{
Issues in Cost Effectiveness in Health Care
}

\author{
Kenneth E. Warner, PhD \\ Professor, Department of Public Health Policy and Administration \\ School of Public Health \\ and \\ Senior Fellow, Institute of Gerontology \\ University of Michigan \\ Ann Arbor, MI 48109
}

\begin{abstract}
Cost-effectiveness analysis (CEA) is becoming increasingly popular as society moves toward rationalizing health costs. This review describes the applications and limitations of the technique. Conceptually simple though frequently complicated in application, CEA compares the cost of a procedure with its effectiveness, thus helping an administrator to judge whether the procedure is worth its cost. CEA also permits comparison of various interventions that result in a similar health outcome. A major benefit of CEA is that it forces decision makers to confront the tradeoffs implicit in all decisions regarding alternative approaches. Limitations of the CEA philosophy and technique also have to be understood if it is to be employed effectively; it is not an assessment of cost savings, nor is it a decision-making technique because it does not incorporate value judgments. A number of potential applications to dentistry are described.
\end{abstract}

Key Words: cost-effectiveness analysis, economics of health care, dentistry, dental public health.

In the decade of the 1980s, cost containment and cost effectiveness have become dominant themes-and often misused and abused buzzwords (1)-throughout the broad field of health care. The search for efficiency in the provision of health care services is motivated by the high and rising costs of health care, an activity that currently consumes almost twice the share of gross national product that it claimed as recently as the mid-1960s. Toward the goal of rationalizing the allocation of scarce health care resources, health professionals and economists increasingly have turned to cost-effectiveness analysis (CEA) to assess the efficiency of specific health care interventions. Data on health care CEAs, and on the closely related technique of cost-benefit analysis (CBA), indicate that the growth in interest in these techniques can be fairly characterized as explosive. For the period from 1966 through the end of the 1970s, a colleague and I identified approximately 600 professional publications relating to $\mathrm{CEA}$ and $\mathrm{CBA}$ (2). A recent MEDLINE search revealed almost 5,000 contributions pertain- ing to CEA and CBA from 1966 through early 1989.

The field of dental public health has demonstrated interest in cost containment and cost effectiveness, as well. A predecessor to this workshop, held here more than a decade ago, examined cost-effectiveness issues explicitly (3); a 1980 conference, focusing on the cost of dental care, adopted the principle of encouraging dental public health professionals and economists to work together on issues of dental care efficiency (4). Application of CEA and CBA to dental health more generally is found in some 145 publications related directly to this subject, identified during the aforementioned MEDLINE search.

At the outset of this ambitious workshop, therefore, it seems useful to take a shared, critical look at the technique that will dominate your attention over the next few days. What is cost-effectiveness analysis and what can it do (and what can it not do)? What kinds of problems does one encounter in undertaking CEA? How should one interpret the findings of CEAs? And what are some specific issues and concerns that this group will have to address in its attempt to characterize the cost effectiveness of a variety of dental public health interventions?

Nature and Functions of Cost-effectiveness Analysis

At the outset, it is imperative to emphasize what cost effectiveness does not mean: it does not mean cost saving. A cost-saving intervention is one that brings in more resources than it consumes. A delightful outcome that undoubtedly occurs in some instances, cost-saving interventions are considerably less common in health care than many practitioners of the art want to believe (5). Cost effectiveness is something different. It implies a comparison of the cost of an intervention with its effectiveness. In turn, this comparison permits an assessment, by a decision maker, of whether the health outcome derived from an intervention is worth its cost, or it permits comparison of efficiency across interventions producing similar kinds of health outcomes. In essence, cost-effectiveness analysis aids a decision maker in determining how to attain particular desirable outcomes at least cost or, conversely, how to take a given budget and maximize the desirable outcome achieved through its 
TABLE 1

Steps in Cost-effectiveness Analysis

1. Define problem and objective(s)

2. Identify Alternatives

3. Describe production relationships

4. Identify, measure, and value costs

5. Identify and measure effectiveness

6. Discount future costs and effectiveness

7. Address problems of uncertainty

8. Address issues of equity

9. Present and interpret findings

10. Facilitate transition from analysis to

implementation

deployment.

To the uninitiated, the label "cost-effectiveness analysis" frequently connotes a highly sophisticated analytical technique. While there are numerous CEAs that are elaborate, sophisticated, computer modeling exercises, there are also many simple "back-of-the-envelope" CEAs. Illustrative of the latter is the calculation that each participant in this workshop performed, most likely implicitly, in deciding whether or not to attend. The cost of participating is the loss of a couple of days, which could have been devoted to other productive activities or to leisure. Each participant had to decide whether that cost would be worth the anticipated educational or professional benefit. As this experience suggests, there is nothing inherently complex or confusing in cost-effectiveness analysis. Regardless of its level of sophistication, every CEA requires the analyst to work through the ten basic steps in Table 1. Details on what goes in to each of these analytic steps are discussed by Warner and Luce (2).

The potential value of formal CEAs is frequently misinterpreted. A common perception is that CEA is a decision-making technique, an analytical device that will provide an answer to a policy question. If one adopts this perspective, one will find CEA a disappointment, for the technique is ill-suited to the task of making decisions. Rather, at its best, CEA is useful as a decision-assisting technique. In part, the distinction reflects the technical limitations that pervade analyses; these are discussed below. More fundamentally, the distinction between a decision-making and a decision-assisting role for CEA reflects the essential fact that policy decisions inevitably require value judgments, be they political or social, and value judgments must be made by human decision makers, by politicians, and by bureaucrats. Most of us have a visceral negative reaction to the words politics and bureaucracy. But when one contemplates how a society should allocate public resources, it is difficult to envision a more appropriate, or functional, approach. In this context, one wants to employ analytical techniques that will help to optimize the decision making that occurs within
FIGURE 1

Range of Potential Impacts of CBA-CEA

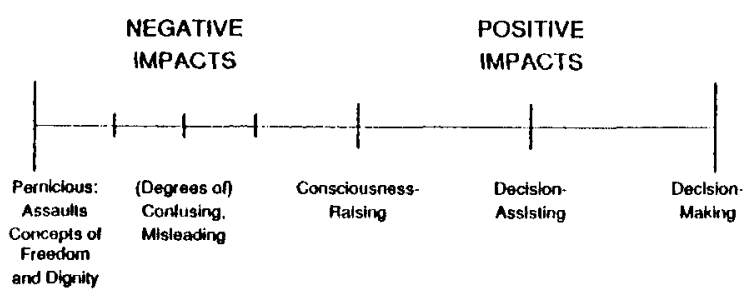

the bureaucratic and political framework.

Figure 1 lends some insight into the spectrum of views of the potential usefulness (or lack thereof) of CEA. On the left-hand side of the spectrum are potential negative outcomes of analysis, possibilities that warranted more attention years ago than they do today. Currently, interest in CEA ranges from the middle perspective (no impact) to the right-hand extreme perception of CEA as a decision-making technique. The points in between the middle and the right-hand end of the spectrum represent what I believe CEA can deliver when it is performed, and interpreted, optimally.

How can CEAs assist in decision making? Done well, they can indeed be consciousness-raising, as Figure 1 suggests, and they can be informative about the quantitative dimensions of problems. CEAs can lend insight into the nature of trade-offs and can force decision makers to confront those trade-offs openly and honestly. They can provide estimates of those dimensions of costs and effectiveness that are properly quantifiable. Best of all, CEAs can provide a framework for discussion of the issues by those affected by interventions, and by those who must decide whether or not to implement them.

\section{Problems in CEA}

Most cost-effectiveness analyses are pervaded by complexities and uncertainties, both conceptual and quantitative. Conceptual issues include, for example, questions about what types of costs or benefits should be considered in a CEA. What opportunity costs should be included in an analysis that are not available through assessments of direct expenditures? This is an obvious issue for this workshop, given that the meeting organizers have only been able to provide estimates of direct programmatic expenditures. What are the social costs involved in dental public health programs? How should analysts deal with the value of patients' time, especially given that patients' time is likely to vary significantly among types of dental public health interventions? (Water fluoridation requires no additional time on the part of patients; a mouthrinse program does, however.) How about the pain and suffering inflicted in the course of an intervention (a cost) or avoided as a result of a successful prevention measure ( $a$ benefit)? What is it 
about good dentition that represents a benefit? Why do we want good dentition? How do we measure it? While we take it for granted that good dentition is desirable, what are the real attributes that we are trying to attain?

In analyzing benefits or effectiveness, there are at least five categories or types of measures that may be of interest. Most prominent among these are personal health benefits, such as good dentition. Health care resource savings constitute a second prominent benefit of interest; these are the dollars that might be saved through an intervention. The three remaining categories are other economic benefits; other social benefits that might be distributional in nature; and intermediate outcomes, such as behavioral change believed to be a precursor of health status improvement (2). As an example of an intermediate outcome, while the ultimate objective of flossing is good dental health, an analyst examining the cost effectiveness of a flossing educational program might measure how many people floss by the end of the program or some time after it ends. The true health benefit "better dental health" would be assumed to follow in some direct proportion.

Quantitative complexities in CEA often seem more mundane than the conceptual issues, but their resolution is of no less importance. In some instances, they reflect conceptual issues directly: if an analyst decides, for example, to include the value of patients' time as a cost variable (a decision regarding a conceptual matter), how should that time be valued (a quantification issue)? While there are standard procedures applied to valuing adults' time (typically at the patients' hourly wage rates), recent analyses have adopted alternative measures (6). In addition, the standard solutions are of little utility in trying to determine how to value the time of schoolchildren taken from their studies to participate in a school-based mouthrinse program.

A common quantitative problem confronting the CEA analyst is the selection of a discount rate. This is the rate at which future years' costs and effectiveness are discounted to convert them to estimates of their present values. An important aspect of cost-effectiveness analysis in general, discounting has particular importance for those interventions that have long-term outcomes, and hence significant lags in the realization of effectiveness (and often costs, as well). A small variation in the discount rate can have significant effects on estimation of cost effectiveness in such circumstances, since both costs and effectiveness are subject to discounting (2).

An issue influencing both the conceptual and quantitative dimensions of a CEA is definition of the perspective of the analysis. CEAs can be approached from three different perspectives. One is the social perspective: $w$ hat is the desirability of an intervention (or the comparative desirability of alternative interventions) from a broad societal perspective? A second perspective is that of an individual who might either be a practitioner or a patient.
Surely, individuals in these categories will view the desirability of an intervention, and its component costs and effectiveness, quite differently. The third perspective, also involving different assessment of costs and effectiveness (their nature as well as measurement), would be that of an organization, be it a health care delivery organization, an insurance company, a governmental agency, or a private business.

The issue of the value of patients' time nicely illustrates how the basic perspective of an analysis affects the identification of categories of costs and benefits and measurement of them. To practitioners, for example, patients' time is of little direct relevance, and hence is a variable that would be omitted from a CEA undertaken from the practitioners' perspective. From a social perspective, by contrast, patients' time may be a significant cost in the equation, as it reflects a true social or opportunity cost. Similarly, patients' time would be included in a CEA reflecting the patients' perspective, although in this case the value of that time would represent a private cost. From an organizational point of view, patients' time might or might not be a relevant cost item, depending on the particular interests of the organization in question. An insurance company might not care about the value of patients' time; a business, on the other hand, would care if interventions were to take place during work time, in which case the business would lose productivity; a union negotiating health care benefits might care whether or not services were received on company time. Note, however, that it might value patients' (i.e., union members') time differently than would the business.

Confronted with a sometimes bewildering array of approaches to CEA, and in particular an absence of clear standards about consistent definition of the components of CEAs and of consistent measurement of them, a few prominent analysts have begun to call for the development of uniform standards so that cost-effectiveness analyses would be directly comparable to one another (7). While the objective is commendable, the call for consistency seems premature at this time. Attainment of a consensus rule or value would be a difficult exercise at best, and one that might lead to consistent but errant analysis. Furthermore, standardization of analytical rules would not accommodate the legitimate differences that reflect differences in perspective. (One proposed "rule" is that all CEAs be undertaken from a social perspective.)

Interpretation of CEAs raises a series of additional questions and problems. Perhaps foremost among these is the issue of generalizability. Is a finding in a clinical setting generalizable to the community setting? This is the standard medical issue of efficacy, or outcome under best-practice conditions, vs effectiveness, outcome in conventional community care (8). Can the results of a small-scale program be extrapolated to a large-scale operation? Will CEA findings generalize from one time 
period to another, when the environment or technology may have undergone considerable change, or from one locale to another with a different institutional setting or labor market (2)?

Finally, in this cursory (and perhaps overly dismal) view of problems in CEA, there is a problem that transcends CEA per se, but seems to pervade this particular analytical endeavor: analyses are often poorly done and yet they are accepted quite uncritically by their readership. This phenomenon is vividly illustrated by a recent survey-based analysis of wellness programs in the workplace setting that concluded: "On average the annual savings [from corporate wellness programs] reported was $\$ 49.74$ per employee....[T]he cost-benefit ratio ... is $\$ 3.44$ saved per dollar of annual operating cost" (9). This figure-indeed, the direct quote-has been cited in scores of speeches and publications extolling the virtues of workplace wellness programs. But consider the origin of the conclusion: 500 companies were surveyed, of which $141(28 \%)$ responded, a response rate typical of commercial studies that would be deemed wholly unacceptable in a scholarly setting because of the likelihood of response bias. Of the respondents, 89 companies (63\%) reported offering wellness programs. Ten companies (11\% of those offering wellness programs) reported having program savings measurements, and three of these ten companies $(30 \%)$ reported their average level of savings. Thus, the often quoted benefit-cost ratio of $\$ 3.44$ comes from self-reports from three companies out of an initial sample of 500 . Aside from the rather poor representation, the survey did not assess how these three companies derived their savings measures.

It is imperative to close this discussion of problems in CEA by emphasizing that, while common, these difficulties do not adhere to all CEAs and certainly not to the best of the art. Well done, with limitations carefully noted, cost-effectiveness analysis can provide a useful quantitative insight into what it will cost to derive a particular set of benefits. More generally, a good analysis, even if it is quite imperfect, can provide perspective on a challenging decision problem. It may be qualitative perspective, as opposed to quantitative perspective, but if it lends insight into the nature of the policy decision choice, it can be immensely helpful.

\section{Nature and Interpretation of Findings in a CEA}

The typical cost-effectiveness analysis yields an aggregated estimate of cost and a single measure of effectiveness. Analysts typically divide the former by the latter to produce a cost-effectiveness ratio. The materials distributed for this workshop focus on the development of such ratios; that is the essential objective of the meeting.

The standard interpretation of cost-effectiveness ratios is that they can be used directly to rank programmatic or policy alternatives in terms of their relative desirability.
Unfortunately, this conventional wisdom is incorrect the majority of the time, even ignoring the aforementioned complexities in defining and measuring costs and effectiveness. The use of the ratios for ranking purposes is valid only if one of two conditions holds: (1) costs are identical across the alternatives, so that one is looking for the highest level of effectiveness given the same level of cost; or (2) effectiveness is the same across the alternatives, in which case one is seeking the lowest costs to attain the given level of effectiveness.

The limitations of the ratio for ranking purposes, as well as the possibilities, are demonstrated in the following example, drawing on the data in Table 2 . This table presents four hypothetical alternative programs: $S, T, U$, and V. For purposes of this example, we assume that the cost figures correctly incorporate all relevant costs, and that the effectiveness measure - number of lives savedaccurately reflects the major health benefits to be derived from the four programs. Thus, we do not confront any of the conceptual or analytical problems raised in the preceding section.

Begin by comparing programs $S$ and $T$. We can make a direct comparison between those two because their costs are identical, $\$ 100,000$. Program T saves more lives than does program $S$. Consequently, T's cost-effectiveness ratio is lower than that of $S, \$ 8,333$ compared with $\$ 10,000$ per life saved. Program T thus clearly dominates program $S$, and because $S$ and $T$ have identical costs, the cost-effectiveness ratio (here a direct reflection of the relative effectiveness of the two programs) is a valid tool for ranking the relative desirability of $S$ and $T$. In a precisely analogous fashion, one sees that program $\mathrm{V}$ dominates program U: each has the same cost; V saves more lives, and consequently produces a lower cost-effectiveness ratio ( $\$ 13,333$ vs $\$ 16,667$ per life saved).

Similarly, we can compare programs $T$ and $U$, because they have identical effectiveness at 12 lives saved. Tbeing less expensive than $U$ leads to a lower cost-effectiveness ratio for $\mathrm{T}(\$ 8,333$ vs $\$ 16,667$ per life saved). The ratio is a valid measure for ranking because the identical effectiveness measures make the ratio rankings the same as a ranking by least cost to achieve the given health outcome.

TABLE 2

Cost-effectiveness Ratio and Ranking of Program Desirability

\begin{tabular}{lccc}
\hline Program & Cost (\$) & $\begin{array}{c}\text { Cost- } \\
\text { (No. of Lives } \\
\text { Saved) }\end{array}$ & $\begin{array}{c}\text { Ratio (\$/Life } \\
\text { Saved) }\end{array}$ \\
\hline S & 100,000 & 10 & 10,000 \\
T & 100,000 & 12 & 8,333 \\
U & 200,000 & 12 & 16,667 \\
V & 200,000 & 15 & 13,333
\end{tabular}


(Note that U has now been "beaten" twice, once on cost grounds, the other time on effectiveness.)

Which of $S, T, U$, and $V$ is the most desirable program? The above logic has reduced the comparison problem to two options: $T$ and $V$. The conventional wisdom would argue that $T$ is the best alternative because its cost-effectiveness ratio is lower than that of $V(\$ 8,333$ vs $\$ 13,333$ per life saved). But in this comparison, neither cost nor effectiveness is the same for the two groups. If the decision makers were restricted to selecting a single program-that is, if they did not have the option of choosing two $\$ 100,000$ programs instead of one $\$ 200,000$ program-would T be clearly preferable to V?

The answer would be unequivocally in the affirmative if one relied uncritically on the cost-effectiveness ratio. But note the implication of doing so: choice of program $T$ instead of $V$ means forgoing the saving of three additional lives in program $V$ in exchange for saving $\$ 100,000$. Is this a "good deal"? Program V saves the additional three lives at a marginal, or extra, cost of $\$ 33,333$ per life saved. Compared with a wide variety of health care interventions, this price for life saving is a veritable bargain (10). As such, the simplecost-effectiveness ratio does not provide a "correct" ranking for programs $\mathrm{T}$ and $\mathrm{V}$.

The issue here is one of a value judgment, one that must be made by a political or bureaucratic decision maker: is the gain of three more lives saved worth the extra expenditure of $\$ 100,000$ ? This question does not lend itself to a neat, mathematical, objective answer. The example illustrates both the utility of cost-effectiveness ratios - they permitted us to narrow the choice set from four to two options-and the futility of relying exclusively and uncritically on this single summary number. (Incidentally, note that with the option of choosing two programs, $S$ plus $T$ certainly would dominate $V$, since the former would save seven more lives collectively $[10+12]$ than would the latter [15]. Still better would be replicating program $\mathrm{T}$, since two "units" of $\mathrm{T}$ would produce 24 lives saved for the $\$ 200,000$. Often, however, an agency does not have the flexibility to pursue more than one programmatic strategy.)

The cost-effectiveness ratio has another implicit limitation, not illustrated by the preceding example: it treats the choice problem as if there is only one nonmonetary outcome worthy of attention. Often this may be the case. A health program may have a single dominant health outcome; water fluoridation may be a good example in this context. Alternatively, analysts may be able to develop an index that captures multiple outcomes in a useful manner. In dental public health, the DMFT measure comes to mind; more generally in health care, a variety of health status indices have been developed, including the most well-known acronym in the field, QALYs, or the index of quality-adjusted life-years (2).

Often, however, real-life health programs may not produce easily indexed outcomes. Commonly, programs
TABLE 3

Arraying Multiple Effectiveness Measures in a CEA

\begin{tabular}{lcccc}
\hline & & \multicolumn{3}{c}{ Saved } \\
\cline { 3 - 5 } Program & Costs (\$) & $\begin{array}{c}\text { Years of } \\
\text { Life }\end{array}$ & $\begin{array}{c}\text { Days of } \\
\text { Morbidity }\end{array}$ & $\begin{array}{c}\text { Days of } \\
\text { Disability }\end{array}$ \\
\hline W & 100,000 & 5 & 25 & 30 \\
X & 100,000 & 5 & 20 & 25 \\
Y & 100,000 & 4 & 400 & 35 \\
Z & 200,000 & 7 & 60 & 80 \\
\end{tabular}

may have multiple outcomes that do not lend themselves neatly to being measured, weighted, and summed into a single index. In such instances, an alternative approach might be to array costs and outcomes in a matrix form. This approach is illustrated in Table 3. Programs $W, X, Y$, and $Z$ are arrayed with their costs and three types of effectiveness outcome measures: years of life saved, days of morbidity avoided, and days of disability avoided. No single cost-effectiveness ratio provides a "bottom line" valuation of the alternative programs. Nevertheless, the matrix summarizes the findings of the CEA and provides considerable useful information to the decision makers who will have to choose among the alternatives.

Note that the data in Table 3 permit some objective narrowing of the choice problem. Specifically, program $W$ clearly dominates program $X$, since it has the same costs and the same or more effectiveness in each of the three outcome categories. Beyond this comparison, however, there is no objective domination among $W, Y$, and $Z$. They all have different attributes; each entails different trade-offs. By arraying the outcomes in this fashion, however, the analyst makes these trade-offs explicit and thereby informs the decision process.

This matrix approach to concluding a cost-effectiveness analysis has been applied very infrequently. $\mathrm{Ob}-$ viously, it is not as satisfying as a single definitive conclusion. Still, it is informative, it can be more comprehensive, and it can be quite useful. This is an approach that one hopes will gain acceptance in the community of health services research.

\section{Dealing with the Ambiguities in CEA}

The uncertainties and complexities found in most CEAs beg attention. Fortunately, there are analytical techniques available to assist in grappling with such problems, and there are reminders of the true potential of CEA that can put these problems in perspective. Notable among these are the following:

1. Liberal use of sensitivity analysis can determine the importance of uncertainties, data problems, and the like. While the term "sensitivity analysis" encompasses several specific techniques (2), all of them share the principal notion: vary the uncertain element and find out 
whether or not it affects the basic qualitative finding of an analysis. Through sensitivity analysis, analysts can learn that a nagging uncertainty is not important, that is, it may not affect the study's basic conclusions. Alternatively, sensitivity analysis can tell an analyst that a particular uncertainty is critical to the conclusion, and thereby recommend caution in interpretation of findings. The latter outcome also implicitly suggests an agenda for further research to narrow the important uncertainties.

2. Of great importance is a constant reminder, to analyst and decision-making client alike, that CEA's legitimate role is to contribute information and perspective to a decision problem that is inherently subjective. As such, the limitations of CEA are appreciated in context, and the utility of analysis perhaps better understood.

3. Finally, both analysts and decision makers need to recognize that progress is being made in refining the technical art of CEA. The methodology of analysis has witnessed several improvements over the past decade and more can be anticipated in the future. We need not await such improvements to improve the practice of CEA today, however. Efforts need to be made to get analysts to incorporate existing state-of-the-art methodology into their work. Altogether too many CEAs are weak because of poor application of good techniques, rather than the reverse.

In his foreword to our textbook on cost-benefit and cost-effectiveness analysis in health care (2), health economist Victor Fuchs concluded that we were unduly cynical about the potential of CBA and CEA to find useful niches in the world of health care policy decision making. In less than a decade since the book's publication, these techniques have found more application in the health policy forum than we anticipated. As such, we find that Fuchs' admonition to our readers bears emphasis: cynicism reflecting the limitations of CEA should be balanced with optimism about the contribution analysis can make to hard policy choice problems.

\section{CEA and this Workshop: Specific Thoughts}

Given the focused objective of this workshop, it may be useful to conclude with some observations directly related to the task that lies ahead. Some are general in nature, others quite specific to challenges participants will confront head on. First and perhaps foremost, it is essential that the workshop not get bogged down in the esoterica of the analysis and in particular in the imperfections of the data that have been provided. Participants will have to remain goal oriented, focused on the bigger picture the workshop ultimately intends to address. As such, individual groups should work through the derivation of the cost-effectiveness data and then, afterward, see how comfortable group members feel with the results. The alternative is to worry about them before they are derived, an approach that will almost certainly fail to produce cost-effectiveness estimates. Indeed, one test of the quality of estimates will be the consistency of findings across groups. While there are good reasons to expect some differences, certain consistencies should emerge if the basic task, and the workshop approach to it, are sensible. This conclusion will not even be testable if all groups do not produce the assigned ratios.

Specific limitations of data and analysis deserving of attention during the course of the workshop include the following:

1. Only direct program costs have been provided to workshop participants. This limitation relates in part to the issue of the perspective of the analysis, discussed below.

2. The data on program costs are likely to be significantly flawed. This reflects both the exigencies of data collection from numerous busy dental health administrators and the fact that even perfect budgetary data need not accurately reflect true program costs (2).

3. Are the effectiveness data provided for this workshop true estimates of effectiveness, reflecting outcomes in conventional practice, or are they really estimates of efficacy, estimating the best that can be achieved, under optimal circumstances (8)? Effectiveness, rather than efficacy, is what is desired for the purposes of this workshop. The next speakers can address this issue with considerable expertise.

4. Effectiveness data pertaining to interventions for the elderly are particularly difficult to find. How will the workshop deal with this challenge?

5. There are going to be differences in economies of scale across the programs. Specifically, water fluoridation likely exhibits significant economies of scale, as is suggested by the data provided. The alternatives that involve treatments for individual patients or clients likely will exhibit something approximating constant returns to scale. Workshopgroups will have to struggle with how to deal with this phenomenon when the objective is to produce single measures of programs' cost effectiveness and rankings of alternatives.

As noted earlier, frequent use of sensitivity analysis is recommended whenever cost or effectiveness estimates vary substantially. For an issue such as the effectiveness of treatments for the elderly, for which data are particularly scarce, break-even analysis may prove to be particularly useful (2).

One major test of the sensitivity of findings relates to different groups' ordinal rankings of program alternatives. Even if specific cost estimates differ across the groups, the relative rankings of program alternatives by cost-effectiveness ratios may not. If this outcome is achieved, the group should feel quite confident about, and satisfied with, the essential findings of the meeting. Note, however, that the lack of consistency in rankings need not indicate that the exercise has failed.

The group as a whole needs to address the issue of the 
perspective of analysis at the outset of the workshop. While most participants might desire to adopt a social perspective, pragmatic considerations, including data availability, may force a narrower public health agency budget perspective. This is not necessarily undesirable; however, the workshop would benefit from explicit discussion about this issue prior to initiating work on specific calculations.

In contemplating the various interventions, participants must be cognizant of the possibility that the cost effectiveness of a procedure will vary depending on the availability of other procedures. For example, in a community with a fluoridated water supply and good brushing habits, a mouthrinse program likely would have a relatively low yield, and hence a high cost-effectiveness ratio. In a community lacking fluoridated water, and in which good brushing practices may be less common, a mouthrinse program would be expected to have greater effectiveness, and hence better cost effectiveness. This observation leads to two conclusions: (1) cost-effectiveness ratios are often situation-specific. Analysts must address this in attempts to produce meaningful generalizable results; and (2) estimates of cost effectiveness of this sort provide only partial insight into the desirability of an intervention from the perspective of an individual community. It is possible to imagine, for example, a situation in which the residents of a poor community would find that it would cost them less using a mouthrinse program to reduce caries incidence than it would a more affluent community, and yet the people in that affluent community would want the program, while the people in the poorer community would not. This seemingly anomalous result would reflect differences in the communities' demands for the service. The people in the more affluent community might value good dentition more than the people in the poorer community. The implication is that the generation of cost-effectiveness ratios does not, in itself, provide complete information for the determination of the optimal allocation of dental health care resources. Cost-effectiveness information must be married with community-specific knowledge of demand.

Finally, in struggling with the data imperfections and problems, workshop participants must recognize that one of the purposes of going through this exercise is to determine how much can be learned from it. In other words, the outcome of this meeting is not simply the bottom-line numbers, but rather determination of what a process like this can accomplish. Given the unique structure of the workshop, the success of the process itself ultimately may prove as important as the explicit cost-effectiveness findings. Either way, the assignment is a challenging one, with all the difficulty and interest that the word connotes.

\section{References}

1. Doubilet P, Weinstein MC, McNeil BJ. Use and misuse of the term "cost-effective" in medicine. New Engl J Med 1986 Jan;314:253-6.

2. Warner KE, Luce BR. Cost-benefit and cost-effectiveness analysis in health care: principles, practice, and potential. Ann Arbor, MI: Health Administration Press, 1982.

3. Burt BA, ed. The relative efficiency of methods of caries prevention in dental public health; proceedings of a workshop at the University of Michigan, Ann Arbor, MI, Jun 5-8, 1978.

4. Kudrle RT, Meskin L, eds. Reducing the cost of dental care. Minneapolis, MN: University of Minnesota Press, 1983.

5. Russell LB. Is prevention better than cure? Washington, DC: Brookings Institute, 1986.

6. Hatziandreu EI, Koplan JP, Weinstein MC, Caspersen C], Warner $\mathrm{KE}$. A cost-effectiveness analysis of exercise as a health promotion activity. Am J Public Health 1988 Nov;78:1417-21.

7. Russell LB. Evaluating preventive care: report on a workshop. Washington, DC: Brookings Institute, 1987.

8. Congress of the United States, Office of Technology Assessment. Assessing the efficacy and safety of medical technologies. Washington, DC: Government Printing Office, 1978.

9. Health Research Institute. Corporate wellness programs; 1987 biennial survey results: participant report. Walnut Creek, CA: Health Research Institute, 1987.

10. Morrall JF III. A review of the record. Regulation;1986 Nov-Dec:2534. 